Praxis Forschung Perspektiven

\section{Band 19, Heft 2, Juni 2009}

Verhaltenstherapie und Bildgebung

Herausgeber: Konrad, K. (Aachen); Ehlert, U. (Zürich)

Editorial

76 Bildgebung und Verhaltenstherapie: Chancen und Grenzen

Konrad, K. (Aachen); Ehlert, U. (Zürich)

\section{Übersichtsarbeiten}

78 Neurofunktionelle Bildgebung bei Angststörungen Reinhardt, I. (Aachen); Jansen, A.; Kircher, T. (Marburg)

86 Funktionelle Bildgebung bei chronischen Schmerzerkrankungen: Implikationen für die Therapie Diers, M. (Mannheim)

94 Realtime fMRT: Perspektiven für die klinische Praxis Habel, U.; Mathiak, K. (Aachen)

Kasuistik - Kinder und Jugendliche

104 Visuelle Symptomprovokation bei einem behandlungsnaiven Jungen mit einer schweren Zwangsstörung. Eine Einzelfallstudie mit funktioneller Magnetresonanztomographie Holtkamp, K. (Aachen/Bad Neuenahr); Do Lam, A.T.A. (Aachen/Bonn); Herpertz-Dahlmann, B.; Konrad, K. (Aachen)

\section{Diskussionsforum}

112 Was leistet die funktionelle Bildgebung für die Evaluation und Weiterentwicklung der Verhaltenstherapie? Flor, H. (Mannheim)

Fragebögen und Skalen

114 PASA - Primary Appraisal Secondary Appraisal. Ein Fragebogen zur Erfassung von situationsbezogenen kognitiven Bewertungen Gaab, J. (Zürich)

\section{Vol. 19, Issue 2, June 2009}

\section{Behavior Therapy and Imaging}

Editors: Konrad, K. (Aachen); Ehlert, U. (Zürich)

\section{Editorial}

76 Brain Imaging and Behavior Therapy: Chances and Limitations

Konrad, K. (Aachen); Ehlert, U. (Zürich)

Review Articles

78 Functional Imaging in Anxiety Disorders

Reinhardt, I. (Aachen); Jansen, A.; Kircher, T. (Marburg)

86 Functional Imaging in Chronic Pain: Implications for Therapy

Diers, M. (Mannheim)

94 Real-Time fMRI: Perspectives for Clinical Use Habel, U.; Mathiak, K. (Aachen)

\section{Casuistic Contribution - Children and Adolescents}

104 Visual Symptom Provocation in a Treatment-Naïve Boy with Obsessive-Compulsive Disorder. A Single-Case Study with Functional Magnet Resonance Imaging

Holtkamp, K. (Aachen/Bad Neuenahr); Do Lam, A.T.A. (Aachen/Bonn); Herpertz-Dahlmann, B.; Konrad, K. (Aachen)

Forum

112 Contribution of Functional Imaging to the Evaluation and Development of Behavioral Therapies Flor, H. (Mannheim)

Questionnaires and Scales

114 PASA - Primary Appraisal Secondary Appraisal. A Questionnaire for the Assessment of Cognitive Appraisals of Situations Gaab, J. (Zürich)

\section{KARGER}

Fax +497614520714

Information@Karger.de www.karger.com
(C) 2009 S. Karger GmbH, Freiburg 


\section{Band 19, Heft 2, Juni 2009}

Interview

118 Guy Bodenmann und Kurt Hahlweg: «Ein individuumszentriertes Störungsverständnis gehört zunehmend der Vergangenheit an» Ditzen, B. (Zürich)

122 Buchbesprechungen

126 Fort- und Weiterbildung

130 Mitteilungen der Verbände

132 Tagungen und Kongresse

121 Impressum

135 Hinweise für Autoren

U2 Erläuterungen zum Titelbild (2. Umschlagseite) Leibl, C.; Veh, C.; Wagner, J. (Prien am Chiemsee)

Einen Ausblick auf den Inhalt der kommenden Hefte finden Sie auf Seite 136.

\section{Vol. 19, Issue 2, June 2009}

Interview

118 Guy Bodenmann and Kurt Hahlweg: 'A Concept of Disease That Centers Only around the Individual Has Become a Story from the Past' Ditzen, B. (Zürich)

122 Book Reviews

126 Education

130 Information by Behavior Therapy Associations

132 Meetings and Conferences

121 Imprint

135 Guidelines for Athors

C2 Explanations on the Title (Inside front cover) Leibl, C.; Veh, C.; Wagner, J. (Prien am Chiemsee)

Forthcoming papers are listed on page 136. 